\title{
A 9-Year-Old Female With a Cough and Cavitary Lung Lesion
}
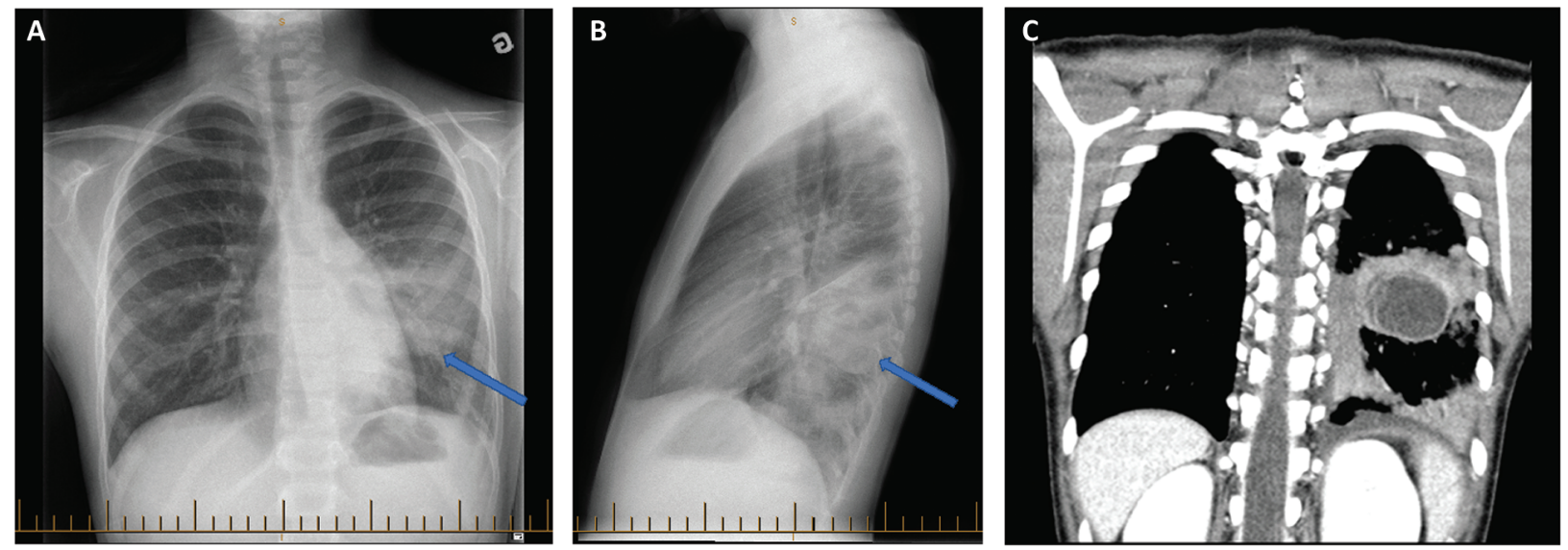

Figure 1. Posteroanterior $(A)$ and lateral $(B)$ chest $X$-ray and coronal computed tomography $(C)$ of the thorax with intravenous contrast demonstrating a fluid-filled collection $(A-C)$, with a thickened rim and double-contour within the left lower lobe $(C)$. There are marked surrounding consolidative changes involving the left lower lobe $(A-C)$.

A 9-year-old female from central Quebec, Canada, presented to a community hospital with 48 hours of chest pain and dyspnea, acutely worsening in the preceding 12 hours, superimposed on a 3-week history of intermittent cough, rhinorrhea, and sore throat. Her initial examination was remarkable for left-sided rales on auscultation. A chest X-ray revealed a left lower lobe cavity (Figure 1A and 1B). Subsequent computed tomography (CT) demonstrated a $3.5 \times 3.8 \times 3.1 \mathrm{~cm}$ left lower lobe cavitary lesion with an air-fluid level and thickened rim with surrounding consolidation (Figure 1C). She was given a presumptive diagnosis of bacterial lung abscess, started on broad-spectrum antibiotics, and transferred to our center for evaluation.

On arrival, she appeared fatigued and pale, but her physical examination was otherwise unremarkable. The white blood cell count was $14.2 \times 10^{9}$ cells $/ \mathrm{L}$, with elevated eosinophils $\left(1.6 \times 10^{9}\right.$ cells/L). The remainder of her laboratory investigations, including renal function, electrolytes, and liver enzymes, were within normal limits. After admission, she was intermittently febrile with a maximum recorded temperature of $39.9^{\circ} \mathrm{C}$.

What is your diagnosis? 
Anthony Amoroso and Ajit P. Limaye, Section Editors

\section{A 9-Year-Old Female With a Cough and Cavitary Lung Lesion}

Diagnosis: Ruptured hydatid cyst of the lung.

The differential diagnosis for the patient's presentation was broad and included bacterial lung abscess, superinfected congenital cystic adenomatoid malformation, superinfected bronchogenic cyst, mycobacterial and fungal infections, as well as parasitic diseases such as paragonimiasis and cystic echinococcosis (CE). The clinical presentation coupled with the peripheral blood eosinophilia and the CT imaging were suggestive of a diagnosis of ruptured echinococcal cyst. Review of her thoracic CT scan demonstrated the additional presence of 2 small cysts within her liver. A liver ultrasound revealed 1 cyst in segment $4(2.1 \times 2.7 \times 2.0 \mathrm{~cm})$ and 1 in segment $8(1.6 \times 1.6 \times 1.7 \mathrm{~cm})$, staged as CE2 and CE1, respectively (Figure 2), according to the WHO-IWGE (World Health Organization-Informal Working Group on Echinococcosis) ultrasound classification of hydatid liver disease [1]. Additional history was obtained. The patient frequently accompanied her father on hunting trips in their region. Their game, which included moose and deer, would be butchered in the field, and frequently their accompanying dog would be allowed to consume the offal.

An in-house developed serum enzyme-linked immunosorbent assay using crude antigens for Echinococcus granulosus was positive with an optical density (OD) of 2.48 (OD cutoff value, 0.35 ), further supporting the diagnosis of hydatidosis [2, 3]. Albendazole and praziquantel were initiated, and she underwent left lower lobe lobectomy 6 days later (10 days after the acute onset of dyspnea and chest pain). Post-operatively, her peripheral blood eosinophilia rose to $13.17 \times 10^{9}$ cells/L but then steadily declined to the normal range by 6 weeks after surgery.

Histopathology of the resected lung tissue demonstrated a necrotic and hemorrhagic cavity, extending to the pleura, with abundant eosinophils, easily identifiable lamellar membranes (Figure 3A), and numerous protoscolices with visible hooklets (Figure 3B). Polymerase chain reaction followed by genotyping of the resected cyst revealed $E$. granulosus complex genotype 8 (G8), now recognized as Echinococcus canadensis (genotypes G8 and G10) [4]. Albendazole and praziquantel were continued, and she was discharged from hospital. Her parents and siblings were screened for infection with chest X-rays and liver ultrasounds, but none were found to have evidence of echinococcosis. The family dog was evaluated by a veterinarian and treated with praziquantel, though no tapeworm infection was identified.

Cystic echinococcosis (CE) is caused by infection with the larval form of the cestode Echinococcus granulosus sensu lato
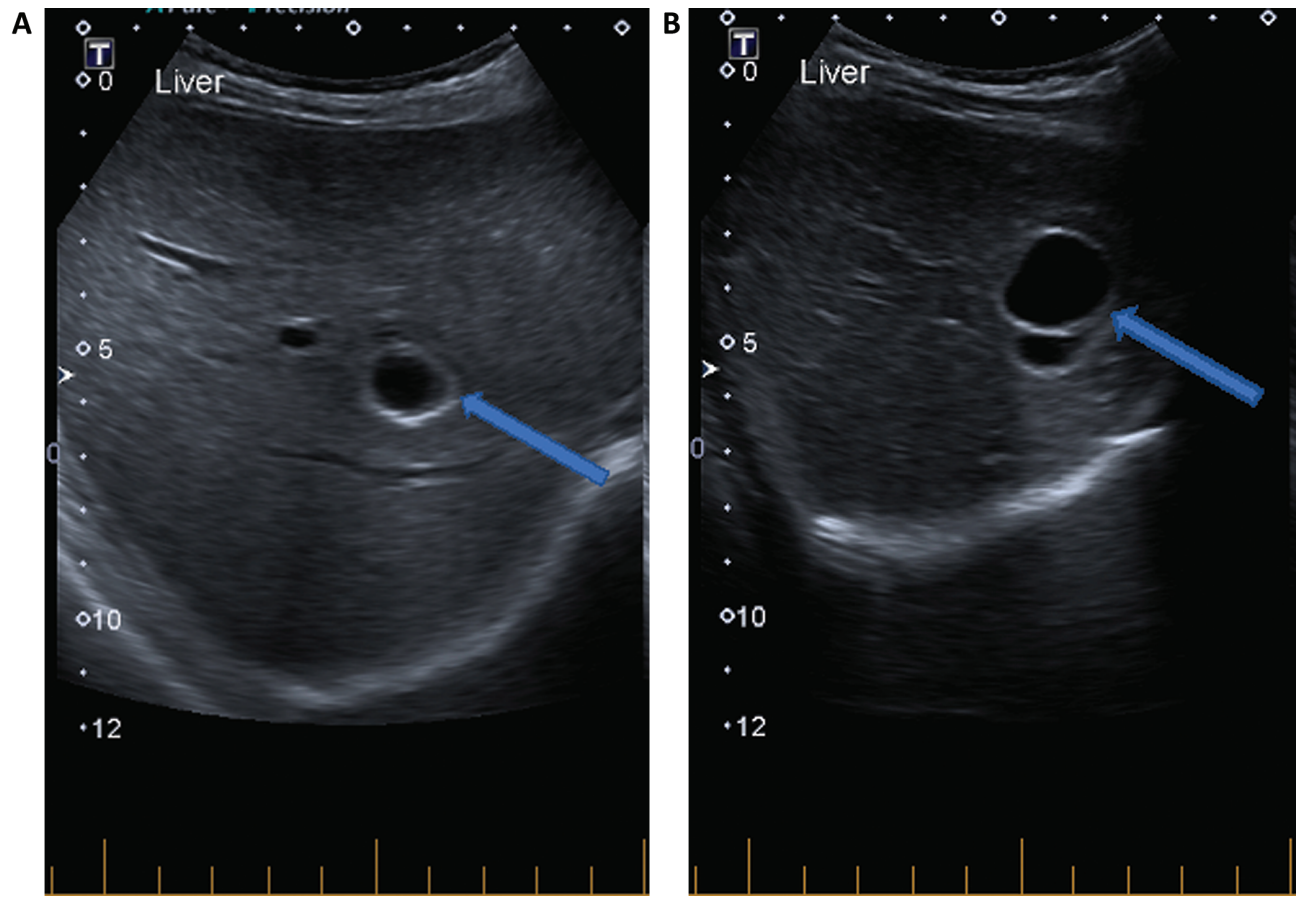

Figure 2. Ultrasound imaging of the 2 liver cysts, demonstrating a simple CE1 cyst (A) and a CE2 cyst (B). 

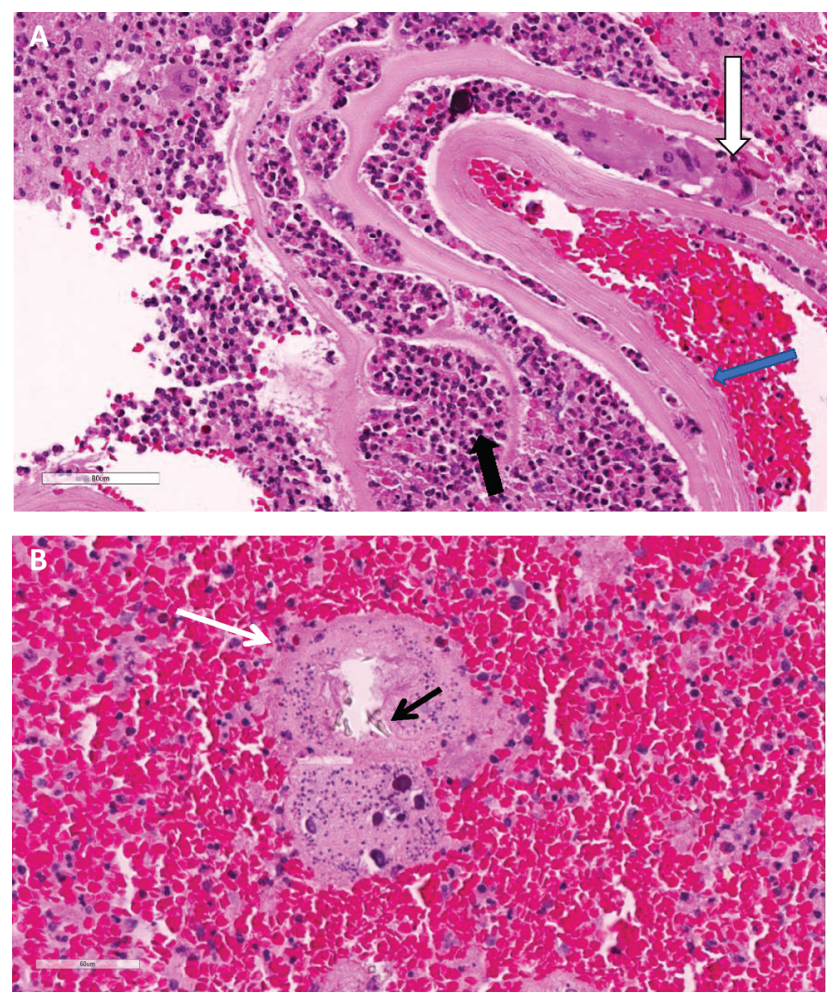

Figure 3. Hematoxylin and eosin staining of histopathology section showing (A) laminated membrane (blue arrow) surrounded by eosinophils (black arrow) with giant cell granulomatous inflammation (white arrow), scale bar: $80 \mu \mathrm{m}$, and $(B)$ partial section through the protoscolex (white arrow) with parts of a rostellum and a parenchymatous portion, plus hooklets (black arrow), scale bar: $60 \mu \mathrm{m}$.

(s.l.). This infection, acquired through the ingestion of eggs shed in the stools of the definitive canine hosts, manifests with the formation of cysts within various tissues, commonly the liver and lung. These cysts grow progressively and, as a result of mass effect, cause local discomfort or even compromised organ function [5]. Growth rate varies according to parasite genotype and host factors such as age, immune function, and tissue elasticity. Pulmonary cysts usually reach $1-2 \mathrm{~cm}$ in diameter at the end of 6 months and up to $6 \mathrm{~cm}$ in diameter within 1 year [6-8]. The cysts can spontaneously rupture, which can be associated with the development of anaphylactoid reactions and other local or systemic complications depending on the site of rupture [9].

Diagnosing echinococcosis can be challenging, as serology may have poor sensitivity, particularly for extrahepatic cysts [10, 11], and eosinophilia is nonspecific and usually absent [12]. In this case, the presence of eosinophilia as well as the strongly positive serology may have resulted from significant antigen spillage due to cyst rupture. As seen in Figure 1C, the presence of a second rind within the cyst was suggestive of a detached endocyst. The finding of liver cysts with characteristic features can confirm the diagnosis in the setting of exposure history. Whereas E. granulosus sensu stricto (genotypes G1-G3) predominate in subtropical and tropical regions, genotypes G8 and G10 are endemic in
North America [4]. Although no human infection with G8 has been reported from Quebec, recent data suggest that it now predominates among wolves and coyotes in eastern Canada [13].

The treatment of echinococcosis varies by stage and location [14]. Surgical resection is considered the standard of care in extrahepatic hydatid disease, though case reviews of patients have suggested that the sylvatic biotype is less virulent than pastoral strains, and a nonsurgical approach is possible for uncomplicated cases [4]. The role of treatment with benzimidazole therapy before surgery remains controversial, with some evidence that it may increase the risk of rupture $[15,16]$. In the case presented here, the patient's acute worsening, parenchymal infiltrate, and peripheral eosinophilia were suggestive that rupture had already occurred. Consequently, albendazole and praziquantel were initiated preresection. Praziquantel was added to albendazole to increase the protoscolicidal effect. This strategy is based on observational studies that suggest improved cure rates without significant adverse events [17-19]. As rupture of hydatid cysts increases the likelihood of recurrence, prolonged treatment and long-term follow-up are planned.

Hydatid cyst of the lung can mimic a bacterial lung abscess and is an important consideration for all patients found to have cavitary lung lesions originating from endemic areas, including southern Canada.

\section{Note}

Potential conflicts of interest. All authors: No reported conflicts of interest. All authors have submitted the ICMJE Form for Disclosure of Potential Conflicts of Interest. Conflicts that the editors consider relevant to the content of the manuscript have been disclosed.

Alexander Lawandi, ${ }^{1, a}$ Cedric P. Yansouni, ${ }^{1,2, a}$ Michael Libman
Earl Rubin, ${ }^{3}$ Sherif Emil, ${ }^{4}$ Chantal Bernard, ${ }^{5}$ Momar Ndao, ${ }^{2,6}$ and Sapha Barkati ${ }^{1,2, a}$,
${ }^{1}$ Division of Infectious Diseases, Department of Medical Microbiology, ${ }^{2}$ J.D. MacLean Centre
for Tropical Diseases, ${ }^{3}$ Division of Infectious Diseases, Department of Medical Microbiology,
The Montreal Children's Hospital, ${ }^{4}$ Division of Pediatric General and Thoracic Surgery, The
Montreal Children's Hospital, ${ }^{5}$ Department of Pathology, and ${ }^{6}$ National Reference Centre for
Parasitology, Research Institute, McGill University Health Centre, Montreal, Quebec, Canada

\section{References}

1. Working Group WHOI. International classification of ultrasound images in cystic echinococcosis for application in clinical and field epidemiological settings. Acta Tropica 2003; 85:253-61.

2. Himsworth CG, Jenkins E, Hill JE, et al. Emergence of sylvatic Echinococcus granulosus as a parasitic zoonosis of public health concern in an indigenous community in Canada. Am J Trop Med Hyg 2010; 82:643-5.

3. Coltorti EA. Standardization and evaluation of an enzyme immunoassay as a screening test for the seroepidemiology of human hydatidosis. Am J Trop Med Hyg 1986; 35:1000-5.

4. Davidson RK, Lavikainen A, Konyaev S, et al. Echinococcus across the north: Current knowledge, future challenges. Food and Waterborne Parasitology 2016 4:39-53.

5. Moro P, Schantz PM. Echinococcosis: a review. Int J Infect Dis 2009; 13:125-33.

6. Balikian JP, Mudarris FF. Hydatid disease of the lungs. A roentgenologic study of 50 cases. Am J Roentgenol Radium Ther Nucl Med 1974; 122:692-707.

7. Halezeroglu S, Celik M, Uysal A, Senol C, Keles M, Arman B. Giant hydatid cysts of the lung. J Thorac Cardiovasc Surg 1997; 113:712-7.

8. Sarkar M, Pathania R, Jhobta A, Thakur BR, Chopra R. Cystic pulmonary hydatidosis. Lung India 2016; 33:179-91.

9. Vuitton DA. Echinococcosis and allergy. Clin Rev Allergy Immunol 2004 26:93-104. 
10. Barnes TS, Deplazes P, Gottstein B, et al. Challenges for diagnosis and control of cystic hydatid disease. Acta Trop 2012; 123:1-7.

11. Manzano-Román R, Sánchez-Ovejero C, Hernández-González A, Casulli A, Siles-Lucas M. Serological diagnosis and follow-up of human cystic echinococcosis: a new hope for the future? Biomed Res Int 2015; 2015:428205.

12. Zhang W, Li J, McManus DP. Concepts in immunology and diagnosis of hydatid disease. Clin Microbiol Rev 2003; 16:18-36.

13. Schurer JM, Bouchard E, Bryant A, et al. Echinococcus in wild canids in Québec (Canada) and Maine (USA). PLoS Negl Trop Dis 2018; $12: \mathrm{e} 0006712$.

14. Brunetti E, Kern P, Vuitton DA; Writing Panel for the WHO-IWGE. Expert consensus for the diagnosis and treatment of cystic and alveolar echinococcosis in humans. Acta Trop 2010; 114:1-16.

15. Sheikhy K, Shadmehr MB. Hemoptysis as a complication of capitonnage for management of pulmonary hydatid cyst. Tanaffos 2014; 13:46-8.

16. Sheikhy K, Abbasidezfouli M, Daneshvar Kakhaki A, Saghebi SR, Malekzadegan A. Different and unpredictable clinical outcome of ruptured pulmonary hydatid cysts. Tanaffos 2015; 14:217-21.

17. Cobo F, Yarnoz C, Sesma B, et al. Albendazole plus praziquantel versus albendazole alone as a pre-operative treatment in intra-abdominal hydatidosis caused by Echinococcus granulosus. Trop Med Int Health 1998; 3:462-6.
18. Haralabidis S, Diakou A, Frydas S, et al. Long-term evaluation of patients with hydatidosis treated with albendazole and praziquantel. Int J Immunopathol Pharmacol 2008; 21:429-35.

19. Alvela-Suárez L, Velasco-Tirado V, Belhassen-Garcia M, et al. Safety of the combined use of praziquantel and albendazole in the treatment of human hydatid disease. Am J Trop Med Hyg 2014; 90:819-22.

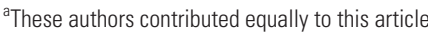

Correspondence: S. Barkati, J.D. MacLean Centre for Tropical Diseases at McGill University, 1001 Boulevard Decarie, Montreal, Quebec H4A 3J1, Canada (sapha.barkati2@mcgill.ca).

\section{Clinical Infectious Diseases ${ }^{\circledR} \quad$ 2019;69(4):705-8}

(C) The Author(s) 2018. Published by Oxford University Press for the Infectious Diseases Society of America. All rights reserved. For permissions, e-mail: journals.permissions@oup.com. DOI: 10.1093/cid/ciy769 